Medicine, College of Medical and Dental Sciences, University of Birmingham, Birmingham, UK

\subsection{6/thoraxjnl-2013-204457.103}

Ageing is associated with increased episodes of sepsis and poorer outcomes. Statins are associated with improved outcomes during infection. We aimed to characterise the impact of age and acute severe infection on key neutrophil functions, assess whether physiologically relevant doses of simvastatin altered neutrophil functions and if benefits were seen, when during a septic episode statins could be utilised.

Methods Neutrophils from healthy volunteers and patients with lower respiratory tract infections (LRTI), pneumonia and sepsis were assessed for migratory accuracy, phagocytosis and neutrophil extracellular trap production before and after in-vitro treatment with simvastatin. Healthy elderly volunteers received $80 \mathrm{mg}$ simvastatin or placebo in a cross over double-blind randomised controlled trial and neutrophil functions were assessed. Data presented is for migration.

Results Neutrophils from healthy subjects ( $\mathrm{n}=70$, aged 21-94) demonstrated preserved neutrophil movement) $\left(\mathrm{R}^{2}=-0.48, \mathrm{p}<\right.$ 0.0001 ) towards chemoattractants (data shown for IL-8). Neutrophil chemotaxis decreased after 60yrs (comparing $<35$ to $>65 \mathrm{yrs}$ : mean difference (MD) $1.25 \mu \mathrm{m} / \mathrm{min}, \mathrm{p}=0.02$ ).

There was a progressive decrease in neutrophil chemotaxis in old patients with a LRTI, pneumonia and severe sepsis (MD compared to healthy control; LRTI $(\mathrm{n}=10), 0.7 \mu \mathrm{m} / \mathrm{min}$, $\mathrm{p}=0.04$; pneumonia $(\mathrm{n}=5), \mathrm{MD} 1.1 \mu \mathrm{m} / \mathrm{min}, \mathrm{p}=0.02$; sepsis $(\mathrm{n}=22) \mathrm{MD} 1.6 \mu \mathrm{m} / \mathrm{min}, \mathrm{p}=0.01)$ with "septic neutrophils" unable to mount targeted chemotaxis. Improvements to baseline were seen following recovery.

In-vitro treatment of neutrophils from healthy older people with simvastatin $(1 \mu \mathrm{M})$ restored "old" neutrophil chemotaxis to that of "young" cells. Simvastatin also restored neutrophil migration from old patients with LRTI and pneumonia to baseline but not in patients with sepsis.

Two weeks of oral simvastatin $80 \mathrm{mg}$ once daily therapy in healthy old volunteers (Age $>65, \mathrm{n}=20$ ) increased the accuracy of neutrophil migration $(\mathrm{MD} 1.68 \mu \mathrm{m} / \mathrm{min}, \mathrm{p}=0.02)$ replicating benchwork.

Conclusions "Elderly" neutrophil function is compromised in health, and deteriorates during infective episodes, in accordance with the severity of the insult. Migratory accuracy can be improved with simvastatin therapy however neutrophil function

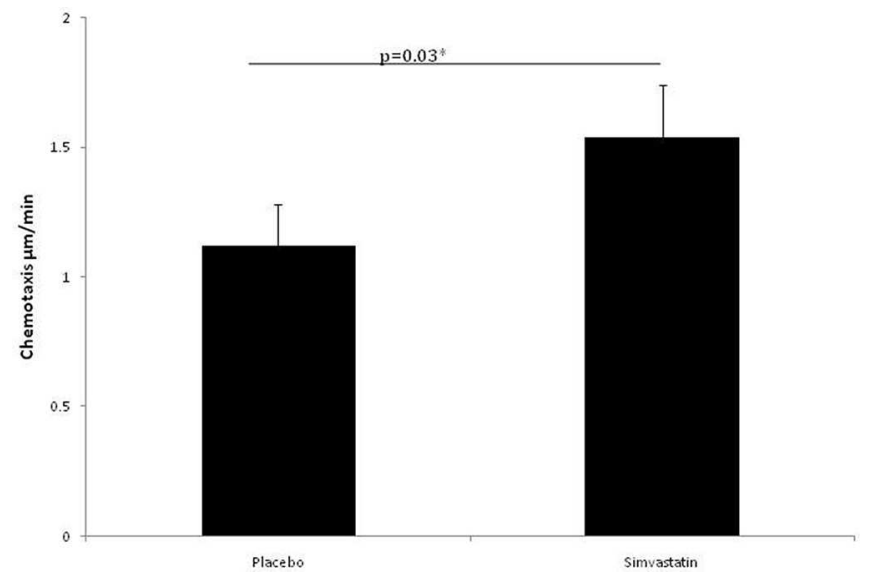

Abstract S96 Figure 1. Simavastatin 80mg once daily for 14 days improves directional migration (chemostaxis) of neutrophils from healthy elderly volunteers towards IL-8. *Student's t-Test in sepsis patients cannot be modulated during short term in-vitro therapy. Our data suggest statin therapy might be a preventative or an early adjuvant intervention rather than a treatment in established sepsis. We are testing whether simvastatin $80 \mathrm{mg}$ for seven days modifies neutrophil responses in elderly patients with pneumonia and sepsis (SNOOPI Trial).

\section{S97 ALVEOLAR EPITHELIAL DNA DAMAGE, INFLAMMATION AND ALTERED AUTOPHAGY FOLLOWING EXPOSURE TO SILVER NANOPARTICLES IS EXACERBATED BY VIRAL LIGANDS IN VITRO}

MZ Zambianchi, TD Tetley, AJ Thorley; Imperial College, London, England

\subsection{6/thoraxjn-2013-204457.104}

Rationale Over the past decade there has been a rapid increase in the development and use of engineered nanoparticles. Silver (Ag) is the most commercialised nanomaterial in the world. Due to its antibacterial activity it is incorporated into a variety of consumer goods including silver nanoparticle sprays to treat pulmonary infection. However, little is known about silver nanoparticle (AgNPs) toxicity in the lung. We hypothesise that inhalation of AgNPs will trigger a pro-inflammatory response in the alveolar epithelium, which, in the presence of viral infection, will synergise with the innate immune response, leading to increased pulmonary epithelial inflammation, DNA damage and autophagy activation.

Methods Transformed human alveolar epithelial type-1-like cells (TT1) were exposed to AgNPs alone and in combination with Poly I:C, a synthetic analogue of double strand RNA that mimics viral infection. Oxidative stress level was measured by dihydroethidium staining. Levels of IL-6 and IL- 8 were assessed by ELISA. DNA damage and autophagy marker LC3II/LC3I ratio were measured by western blot.

Results AgNPs induced oxidative stress in TT1 cells leading to enhanced inflammation, DNA double strand breakage and autophagy activation. The combination of $\mathrm{Ag}$ and Poly I:C potentiated IL-6 release (4-fold; $p<0.01$ ) and DNA damage (3fold; $\mathrm{p}<0.01$ ). Autophagy flux, activated by AgNPs alone, was slowed down by combined AgNPs and Poly I:C exposure.

Conclusion This study shows that Ag could induce oxidative stress in the lung, leading to a strong pro-inflammatory response and DNA damage, both potentiated by co-exposure to Poly I:C. Also, defective autophagy might result in certain human diseases such as cancer and neurodegenerative disease. This suggests that inhalation of AgNPs may have adverse health effects on the lung, that might be exacerbated by viral infection.

\section{S98 VITAMIN D DEFICIENCY INCREASES BACTERIAL LOAD IN} A MURINE MODEL OF SEPSIS-INDUCED LUNG INJURY

${ }^{1} \mathrm{D}$ Parekh, ${ }^{2} \mathrm{RCA}$ Dancer, ${ }^{2} \mathrm{~S}$ Lax, ${ }^{1} \mathrm{GD}$ Perkins, ${ }^{2} \mathrm{DR}$ Thickett; ${ }^{1}$ University of Warwick, Warwick, UK; ${ }^{2}$ University of Birmingham, Birmingham, UK

\subsection{6/thoraxjnl-2013-204457.105}

Introduction We have previously shown that patients with Acute Lung Injury (ALI) are severely vitamin D deficient. Several studies have reported a high prevalence of vitamin D deficiency in critically ill patients with sepsis, associated with increased morbidity and mortality but whether this is cause or effect is unknown. Bacteraemic sepsis is more common in the winter 